Research Article

\title{
The Three-Stage Integrated Optimization of Automated Container Terminal Scheduling Based on Improved Genetic Algorithm
}

\author{
Yiqin Lu (iD) \\ School of Economics \& Management, Shanghai University of Electric Power, Shanghai, China \\ Correspondence should be addressed to Yiqin Lu; lu_yiqin@163.com
}

Received 26 May 2021; Revised 17 September 2021; Accepted 6 October 2021; Published 21 October 2021

Academic Editor: José António Fonseca de Oliveira Correia

Copyright ( 2021 Yiqin Lu. This is an open access article distributed under the Creative Commons Attribution License, which permits unrestricted use, distribution, and reproduction in any medium, provided the original work is properly cited.

\begin{abstract}
This paper proposes an improved GA (genetic algorithm)-based integrated optimization of automated container terminal scheduling. The three-stage integrated optimization model of automated container terminal scheduling is suggested, and the objective is the minimal operation time of the loading and unloading tasks at the automated container terminal. To solve the difficult combination problem, an improved GA, which is named PGA (Probability Genetic Algorithm), is developed. As traditional GA does not change the probability according to the specific iterations in the population, PGA improves the above limitation to improve population distribution and accelerate convergence. Different from published literature, the study of this paper can be presented in two aspects. One is in the modeling; it includes (i) formalizing the description of the purpose of the model and (ii) having a real-world coordination of three types of equipment that are incorporated at automated container terminals. The other is that PGA is applied to deal with the integrated scheduling whose results can be gotten with better solving speed and convergence. Numerical experiments show that the model constructed in this paper has important reference value for the optimum ratio of QCs, AGVs, and ASCs in automated container operation, which is of great significance to improve the efficiency of the automated terminal. Furthermore, compared with the results of traditional GA and PSO (particle swarm optimization), the speed and convergence of PGA have been greatly improved.
\end{abstract}

\section{Introduction and Literature Review}

In order to improve efficiency and profits, traditional container terminals began to use automated technology and stepped into the process of automation construction. Scheduling plays a crucial role in manufacturing and service industries [1]. Improving the operation efficiency of automated container terminals is an urgent problem to be solved. The operation system of the automated container terminal consists of three parts: loading and unloading operations, horizontal transportation operations, and yard operations. Different operations can be completed by different types of mechanical equipment. Different choices of equipment make up different operation schemes of the automated container terminal scheduling. This research is based on the background of "Double-Trolley Quay Cranes (QCs) + Automatic Guide Vehicles (AGVs) + Automatic Stacking Cranes (ASCs)" loading and unloading system of Shanghai automated container terminal at Yang Shan Port. The threelevel integrated scheduling optimization model of the automated container terminal operation system is established, and an improved GA is designed to solve the problem in this paper. The objective is minimizing the completion time of all the tasks, called the makespan, subject to the constraints. The optimization operation scheme formed in this paper can provide research support to optimize the automated terminal scheduling.

My research is the basic problem and hot issue in the field of automation container terminal system. It is worth pointing out that research in this field at home and abroad is still in the early phase. At present, most researches are directed at traditional container terminals and few studies have been done on the automated container terminal scheduling. Studies on the optimization of terminal operations are mainly focused on the following two aspects: 
(1) Research on the optimization of traditional container terminal scheduling: previous studies have mostly focused on the optimization of traditional container terminals, which also has a good reference for this paper. In traditional researches, there were many studies on scheduling of common container terminals, most of which concentrated on the operations of QCs, Yard trucks (YTs), and Yard Cranes (YCs) separately. Studies of isolated scheduling mainly covered distribution and scheduling of QCs [2-4]. YC allocation and work sequences [5-7] were also studied. Scheduling and path optimization of YTs [8-10] were also studied in traditional researches. The joint optimization models were established for only two types of loading and unloading equipment including joint optimization of QCs-YTs [11], QCs-YCs [12, 13], and YT-YCs $[14,15]$. In addition, since these three operations affect each other, the three-stage integrated optimization of the operation system is the most effective means to improve efficiency from the perspective of system optimization [16-18], which was rare in previous literature.

(2) Research on optimization of automated terminal operation system: recent research on the automated terminal horizontal transportation was mostly concentrated on the scheduling of AGVs [19-22]. To solve the path conflicts for an AGV that may occur in the horizontal transportation of automated container terminal, a control strategy was proposed by adjusting the AGV speed of the path node location [19]. Zaghdoud et al. proposed a method to generate collision-free trajectory of AGVs, which would reduce the completion time of container operation [20]. Xin et al. proposed a hierarchical control structure for integrating AGV interactive scheduling and trajectory planning [21]. Luo et al. studied the AGV scheduling in the unloading process of automated container terminals, constructed the MIP model according to the integrated unloading problem, and designed an effective solution [22]. The research on joint scheduling optimization of two kinds of operations includes joint scheduling of QC and AGV $[23,24]$ and joint scheduling of AGV and ASC [25]. In order to solve the cooperative scheduling of the AGVs and the rail gantry cranes, the makespan under different conditions was obtained with the changes of AGVs, QCs, and container areas [25].

In order to realize the optimal scheduling of the whole system, Le et al. proposed a new joint scheduling model of QCs, AGVs, and stacking cranes, which took the whole horizontal transportation system of the automated container terminal as the research object [26]. Zeng et al. considered the cooperation problem of terminal transportation equipment and designed a multistage optimization algorithm [27]. Hu et al. considered the coordinated scheduling mechanism among the three devices, proposed the full- degree-of-freedom scheduling problem of integrated shore bridges, stacking cranes, and yard trucks, and established a mixed integer programming model [28], but AGV was not considered in this study. Dkhil et al. proposed the QC-AGVASC integrated model considering the interaction and constraints among various resources of the terminals [29]. The above literature showed that there were few studies on three-stage integrated scheduling of the automated container terminal scheduling. Therefore, research of the loading and unloading equipment and technology mentioned above is not mature enough.

Genetic algorithms (GAs) were developed by Professor Holland of Michigan University in 1969 [30]. It is a process search algorithm which simulates the natural genetic mechanism and biological evolutionism. Although GA has been successfully applied in many fields, it also has its own limitations, for example, poor local search ability, premature convergence, and random walk [31]. These limitations lead to poor convergence performance of the algorithm, which will take a long time to find the optimal solution. As the traditional GA does not change the probability according to the specific iteration of the population. I improved the limitation to get better population distribution and speed up the convergence. Based on studying a lot of optimization algorithms [32-35], I improved the traditional GA and established PGA.

Operations of container terminals are completed by many operation links and each link affects and restricts each other. Most research on terminal scheduling focused on the traditional terminal and little has been done so far on the integrated optimization research of automated container terminal. A few studies on integrated optimization lacked the deep integration of multitypes of equipment. This paper considers the in-depth optimal system scheme for the automatic container terminal operations. Therefore, the threestage integrated optimization system considered in this paper is more comprehensive and has important theoretical and practical significance. Table 1 shows the novelty of this paper compared with the existing research in terms of formulas and methods.

The innovations of this paper are as follows. One is in the modeling; this includes (i) formalizing the description of the purpose of the model and (ii) having a real-world coordination of three types of equipment that are incorporated at automated container terminals. The objective is minimizing the makespan in three working areas at automated container terminals. Compared with the traditional studies which were conducted on optimization of one or two types of equipment, the study of this paper is more scientific. The joint scheduling optimization limited to one or two types of equipment can only achieve the optimal scheduling of the partial operation system rather than that of the entire operating system. As there are conflicts among the optimization objectives of the QCs, AGVs, and ASCs, the threestage research in this paper is more comprehensive and practical. The other is that an improved GA, which is named PGA, is proposed in this paper. The crossover and variation probability in PGA change with the dispersion of the population, which can improve the convergence speed of the 
TABLE 1: Novelty of this paper compared with the existing research.

\begin{tabular}{|c|c|c|}
\hline & Formulas & Methods \\
\hline $\begin{array}{l}\text { Existing } \\
\text { research }\end{array}$ & $\begin{array}{c}\text { Formulas for traditional container terminals lack of the deep integration optimization of } \\
\text { multitype equipment }\end{array}$ & $\begin{array}{l}\text { Traditional intelligent } \\
\text { algorithms }\end{array}$ \\
\hline This paper & Formula on integrated scheduling for fully automated container terminals & Improved GA (PGA) \\
\hline
\end{tabular}

algorithm and prevent it from falling into local optimum. Experiments show that compared with traditional GA and PSO, PGA solves faster and has better convergence.

The paper is organized as follows. The problems are described and formulated in Sections 2 and 3, respectively, and the model is proposed in Section 3. Section 4 presents the analysis of PGA. Numerical experiments and results analysis are shown in Section 5 followed by conclusions in Section 6.

\section{Problem Description and Assumptions}

The automated production control system is used as the "Brain" to control the automated operations of the loading and unloading and transportation equipment at Shanghai automated container terminal of Yang Shan Port. The system consists of three types of equipment, namely, Quay Cranes (QCs), Automated Guided Vehicle (AGVs), and Automatic Stacking Cranes (ASCs). Figure 1 shows the single-container operation process at the automated terminal. The loading and unloading operation is completed by the double-trolley QCs. The main trolley is controlled remotely, and the second trolley works automatically. They interact through the transfer platform. The horizontal transportation is finished by AGVs. The loading and unloading work of the yard is operated by the ASCs which are fully automated. Three types of equipment work together. The seaside ASCs can complete double-container operation. The yard trucks enter the berth, arriving at the designed container area. The landside ASCs complete the unloading operation and go to the container yard. The export operation is carried out by the seaside ASCs. After the AGVs get the container, they go to the bottom of the QCs. The automatic second trolley hoists the containers to the transfer platform. The main trolley lifts the container for loading and unloading operation. Thus, the entire export operation is completed. The import operation is the opposite operation process.

The optimization can be accomplished based on the following four assumptions:

(A1) The main trolley of the QCs unloads the containers to the transfer platform or ships the containers on the transfer platform to container ships. The time spent is only related to the position of the containers on the ship, ignoring the time the QCs spent on the transit platform.

(A2) The QC, AGV, and ASC can only perform one task at a time.

(A3) The locations of containers in the yard and quayside corresponding to all the tasks are known. After the task assignment is completed, the AGV selects the optimal path to complete the transportation task, ignoring traffic problems and jams.

(A4) There is no empty driving for AGVs; that is, the AGV completes a loading mission and then goes to perform an unloading mission. After completing an unloading mission, AGV goes to perform a loading mission.

(A5) The time of the containers in each state is known.

\section{Notations and Model}

The collection parameters are as follows:

$U$ : task set of loading and unloading containers

A: AGV collection

$S$ : QC collection

$D$ : ASC collection

$|U|=n,|A|=k,|S|=m,|D|=j ;|\bullet|$ represents the number of elements in the collection

The decision variables are as follows:

$t_{s u}$ : the time when the QCs numbered " $S$ " begin to perform the " $U$ " container unloading tasks or the time when the QCs numbered " $S$ " complete the " $U$ " container loading tasks.

$t_{\text {sua }}$ : the time when the second trolleys of the QCs numbered " $S$ " load the " $U$ " containers to the AGVs or the time when the " $u$ " containers are unloaded from the AGVs.

$t_{d u 1}$ : the time when the seaside ASCs of the yard numbered " $D$ " begin to unload the " $U$ " containers from AGVs or the time when the " $U$ " containers are loaded onto the AGVs.

$t_{d u 2}$ : the time when the landside ASCs of the yard numbered " $D$ " begin to unload the " $U$ " containers to the designated position or the time when the " $U$ " containers are loaded from the designated position.

$e_{s u}$ : the time taken by the main trolleys of the QCs numbered " $S$ " to unload the " $U$ " containers to the transfer platform or the time taken by the second trolleys to load the " $U$ " containers from the AGVs to the transfer platform (or the reverse tasks). The tasks performed twice in succession are different.

$f_{u}$ : the time taken by AGVs to transport the " $U$ " containers.

$g_{d u}$ : the time taken by the seaside ASCs of the yard numbered " $D$ " to unload the " $U$ " containers to the temporary storage areas or the time taken by the landside ASCs of the yard numbered " $D$ " to load the 


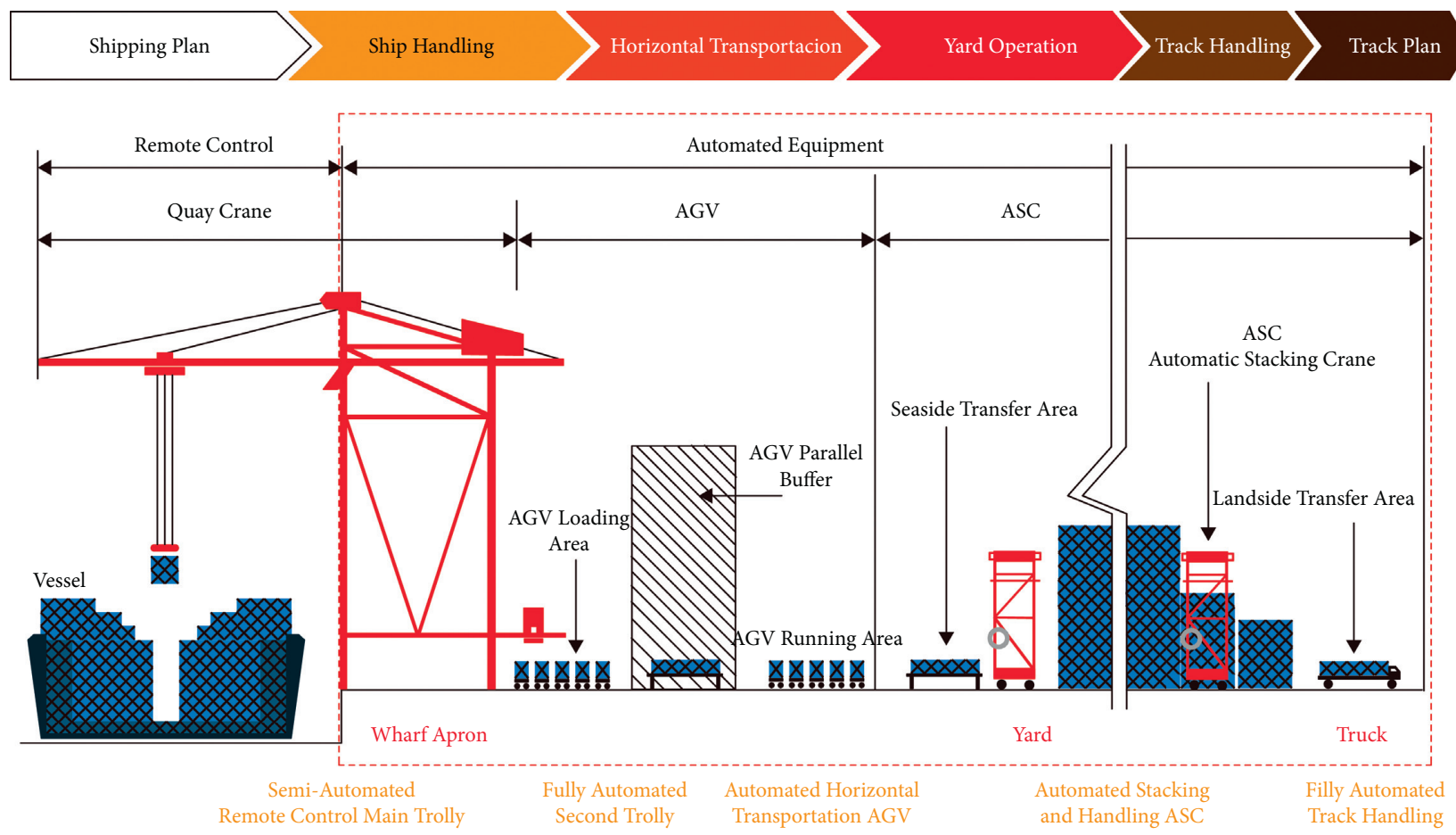

Figure 1: Automated terminal operation flow.

" $U$ " containers from the designated location to the temporary storage area (or the reverse tasks). The tasks performed twice in succession are different.

$\mathfrak{\Im}_{s p}^{s^{\prime} q}$ : it stands whether the same AGV goes to complete the " $q$ " container tasks of the QCs numbered " $s$ " after completing the " $p$ " container tasks of the QCs numbered " $s$ ". If so, $\mathfrak{\Im}_{s p}^{s^{\prime} q}=1$; otherwise, $\mathfrak{\Im}_{s p}^{s^{\prime} q}=0$. Task “ $p$ " and " $q$ " are two types of tasks; that is, " $p$ " is the loading task and " $q$ " is the unloading task or " $p$ " is the unloading task and " $q$ " is the loading task.

$R_{s u}^{a}$ : it stands whether the AGV numbered " $a$ " goes to perform the " $u$ " container task of the QC " $k$." If so, $R_{s u}^{a}=1$; otherwise, $R_{s u}^{a}=0$.

$\aleph_{s u}^{d}$ : it stands whether the " $d$ " yard stores the " $u$ " container of the QC numbered " $k$." If so, $\aleph_{s u}^{d}=1$, otherwise $\aleph_{s u}^{d}=0$.

The model is built according to the parameters set above and takes the minimum time to complete the entire loading and unloading tasks as the objective function. The time of finishing all the tasks is the weighted sum of the time spent in QC work area, the horizontal transport area, and the yard operation area. Since AGV may wait when performing the tasks, each task has a different completion time. The weights of the time spent in QC work area, the horizontal transport area, and yard operating area are respectively determined as $\partial, \eta, \mu$ and the size of the weights is positively correlated with the costs of the operations in the three work areas. In actual operations, the cost of QCs is generally the highest, followed by that of the yard. Cost of AGVs is the lowest. So, there are the following relationships: $\partial>\mu>\eta$.

The whole model is written as follows:

$$
\begin{aligned}
\operatorname{minimize} W= & \partial \sum_{s=1}^{m} \sum_{u=1}^{n}\left|t_{s u a}-t_{s u}\right|+\eta \sum_{d=1}^{j} \sum_{u=1}^{n}\left|t_{d u 1}-t_{s u a}\right| \\
& +\mu \sum_{d=1}^{j} \sum_{u=1}^{n}\left|t_{d u 2}-t_{d u 1}\right|
\end{aligned}
$$

Subject to

$$
\sum_{s^{\natural}=1}^{m} \sum_{q=1}^{n} \mathfrak{\Im}_{s p}^{s^{\prime} q}=1, \quad \forall s \in S, p \in P,
$$

$$
\sum_{a=1}^{k} \mathfrak{R}_{s u}^{a}=1, \quad \forall s \in S, u \in U, a \in A,
$$

$\sum_{u=1}^{n} \mathfrak{R}_{s u}^{a}=1, \quad \forall s \in S, u \in U, a \in A$,

$\sum_{d=1}^{j} \aleph_{s u}^{d}=1, \quad \forall s \in S, u \in U, d \in D$

$\sum_{u=1}^{n} \aleph_{s u}^{d}=1, \quad \forall s \in S, u \in U, d \in D$

$$
e_{s u}<\left|t_{s u a}-t_{s u}\right|, \quad \forall s \in S, u \in U, a \in A,
$$

$t_{s(u+1) a}-t_{\text {sua }}>\left|t_{\text {sua }}-t_{\text {su }}\right|, \quad \forall s \in S, u \in U, a \in A$,

$$
f_{u} \leq\left|t_{d u_{1}}-t_{\text {sua }}\right|, \quad \forall s \in S, u \in U, d \in D, a \in A,
$$




$$
\begin{gathered}
g_{d u}<\left|t_{d u 2}-t_{d u 1}\right|, \quad \forall u \in U, d \in D, \\
t_{d(u+1) 1}-t_{d u 1}>\left|t_{d u 2}-t_{d u 1}\right|, \quad \forall u \in U, d \in D, \\
e_{s u}+e_{s(u+1)} \leq\left|t_{s u a}-t_{s u}\right|, \quad \forall s \in S, u \in U, a \in A, \\
g_{d u}+g_{d(u+1)} \leq\left|t_{d u 2}-t_{d u 1}\right|, \quad \forall u \in U, d \in D, \\
t_{s u a}>0, \\
t_{s u}>0, \\
t_{d u 1}>0, \\
t_{d u 2}>0, \\
e_{s u}>0, \\
f_{u}>0, \\
g_{d u}>0 .
\end{gathered}
$$

Equation (1) is the objective function, which consists of three parts. The first part is the product of the time spent at the QC work area and its corresponding weight. The second part represents the product of the time spent in the horizontal transport area and its corresponding weight. The third part is the product of the time spent in the yard area and its corresponding weight. The sum of the time of the three parts is the objective function of the model, which minimizes the time to complete all container tasks. Equation (2) represents the total loading or unloading tasks performed by an AGV at the same time and defines the sequence of the AGVs to complete container tasks. It indicates that one AGV can only complete the unloading task after completing the loading task of QC or complete the loading task after completing the unloading task of QC. Thus, the empty driving of AGV is avoided. Equation (3) indicates that a container task can only be completed by one AGV. Equation (4) indicates that one AGV can only complete one container task at a time. Equation (5) shows that one container can only be placed in one yard. Equation (6) shows that ASC can only complete one container task at a time. Equation (7) expresses that the time taken for loading and unloading the " $u$ " container to the transit platform is less than the time spent at the QC operating area, because the QC operation consists of two parts, namely, unloading the container from the ship to the transfer platform and unloading the container from the transfer platform to AGV. Equation (8) indicates that the interval between two container tasks is longer than that of one container task at the QC operation area, because the interval between two container tasks is the sum of the time of a container task in three operation areas. Equation (9) shows that the time of transporting a container by AGV is less than or equal to the time of a container at the AGV operation area, because there may be waiting time for $\mathrm{AGV}$ in the transport area. Equation (10) expresses that the time for the " $u$ " container to be transported to the temporary yard area is less than that to the yard area, because yard operation includes two parts, i.e., operating the container to temporary yard area and then to the designated location. Equation (11) indicates that the interval between two container tasks is greater than the time spent on one container task in yard operation area. Because one container task starts only after it is completed, there may be waiting time between the end of the previous task and the beginning of the next task. Equation (12) indicates that the time for the container at QC operation area consists of two parts, namely, the time for the main trolley to load and unload the container to the transfer platform and the time for the second trolley to operate the container to the AGV. Equation (13) indicates that the time of container in the yard operation area is composed of the time for the seaside ASC to operate the container to the temporary yard area and the time for the landside ASC to operate the container to the designated location. Equation (12) indicates that the sum of the time spent by the main trolley in loading and unloading containers to the transfer platform and the time spent by the gantry trolley in loading and unloading containers to the AGV is not greater than that spent in the QC operation areas, because the containers may lose time on the transfer platform. Equation (13) shows that the sum of the time taken by the front ASCs in loading and unloading containers to the temporary storage area and the time spent by the back ASCs in loading and unloading containers to the designated location are not greater than the time spent in the yard operation areas, because the containers may have time loss in the temporary storage area. Equation (14) shows that values of all the decision variables are positive.

\section{Improved GA (PGA)}

4.1. GA Background. GA was inspired by the genetic and evolutionary processes of the biological world and it was the randomized search method based on the survival of the fittest. The basic steps of GA to solve an optimization problem are as follows. (1) Determine the fitness function according to the optimization problem which is generally the objective function. Set the maximum number of iterations and randomly select or generate $N$ individuals as the initial. (2) Determine the individual fitness according to the fitness function. (3) Select individuals with better fitness and generate new individuals by crossover and variation simultaneously. (4) Evolve interactively according to this process until the maximum number of iterations is reached. Finally select the individual with the best fitness as the optimal solution.

4.2. The Improved Crossover and Variation Method. The crossover probability of individual is $P_{a}$ and the variation probability of individual is $P_{b} . P_{a}$ and $P_{b}$ have an important influence to enhance the convergence speed of the algorithm. In the traditional GA, $P_{a}$ and $P_{b}$ were fixed and the probabilities would not change according to the specific iteration in the population. This paper improves the above limitation of the algorithm, that is to say, $P_{a}$ and $P_{b}$ will change according to the dispersion of population fitness so as to improve the population distribution and accelerate the convergence. The formulas are as follows: 


$$
\begin{aligned}
g_{\mathrm{av}} & =\frac{1}{n} \sum_{i=1}^{n} g_{i}, \\
\sigma & =\sqrt{\frac{1}{n}\left(\sum_{i=1}^{n}\left(g_{i}-g_{\mathrm{av}}\right)^{2}\right),} \\
g_{\lim } & =\frac{1}{2}\left(g_{\max }-g_{\mathrm{av}}\right) .
\end{aligned}
$$

Formula (15) represents the calculation of the average value of population fitness and $g_{\mathrm{av}}$ is the average value of the population fitness. $n$ is the number of individuals in the population. $g_{i}$ is the fitness of the " $i$ " individual in the population. Equation (16) expresses the dispersion level of the population fitness by standard deviation. $\sigma$ stands for the dispersion level of population fitness. (17) represents the calculation of the population fitness threshold $g_{\text {lim }} \cdot g_{\max }$ represents the maximum of population fitness.

The improved crossover probability $p_{a}$ and variation probability $P_{b}$ are calculated as follows:

$$
\begin{aligned}
& p_{a}=k_{1} \frac{\sigma}{g_{\lim }}, \\
& p_{b}=k_{2} \frac{g_{\mathrm{lim}}}{\sigma} .
\end{aligned}
$$

$k_{1}$ and $k_{2}$ indicate the fitness factor, which can take different values as needed. When $\sigma<g_{\text {lim }}$, then $\left(\sigma / g_{\text {lim }}\right)<1$, $\left(g_{\lim } / \sigma\right)>1$. It indicates that the value of the population fitness is relatively concentrated. At this time, it is difficult to generate new excellent individuals by crossing and more variations are needed to generate new individuals. So, the variation probability $p_{b}$ should be increased and the crossover probability $p_{a}$ should be reduced. Accordingly, the value of the corresponding fitness factor $k_{1}$ should be reduced and the value of $k_{2}$ should be increased. The value of $k_{1}$ is generally 0.2 and the value of $k_{2}$ is generally 0.8 . Conversely, when $\sigma>g_{\text {lim }}$, then $\left(\sigma / g_{\text {lim }}\right)>1,\left(g_{\lim } / \sigma\right)<1$. It shows that the value of the population fitness is scattered and only crossover is needed to generate new good individuals. Variation should be reduced to prevent damage to good individuals. Therefore, the crossover probability $p_{a}$ should be increased and the variation probability $p_{b}$ should be reduced. Correspondingly, the value of the fitness factor $k_{1}$ should be increased and the value of $k_{2}$ should be reduced. The value of $k_{1}$ is generally 0.8 and the value of $k_{2}$ is generally 0.2 . The above improvement can greatly reduce the running time of the algorithm, speed up the convergence, and prevent the algorithm from falling into a local optimal solution. The algorithm flowchart of PGA is shown in Figure 2.

\section{Numerical Experiments and Results}

This experiment verified the impact on the time to complete tasks by changing the quantity ratios of containers, QCs, AGVs, and ASCs. The quantity ranges are as follows: the

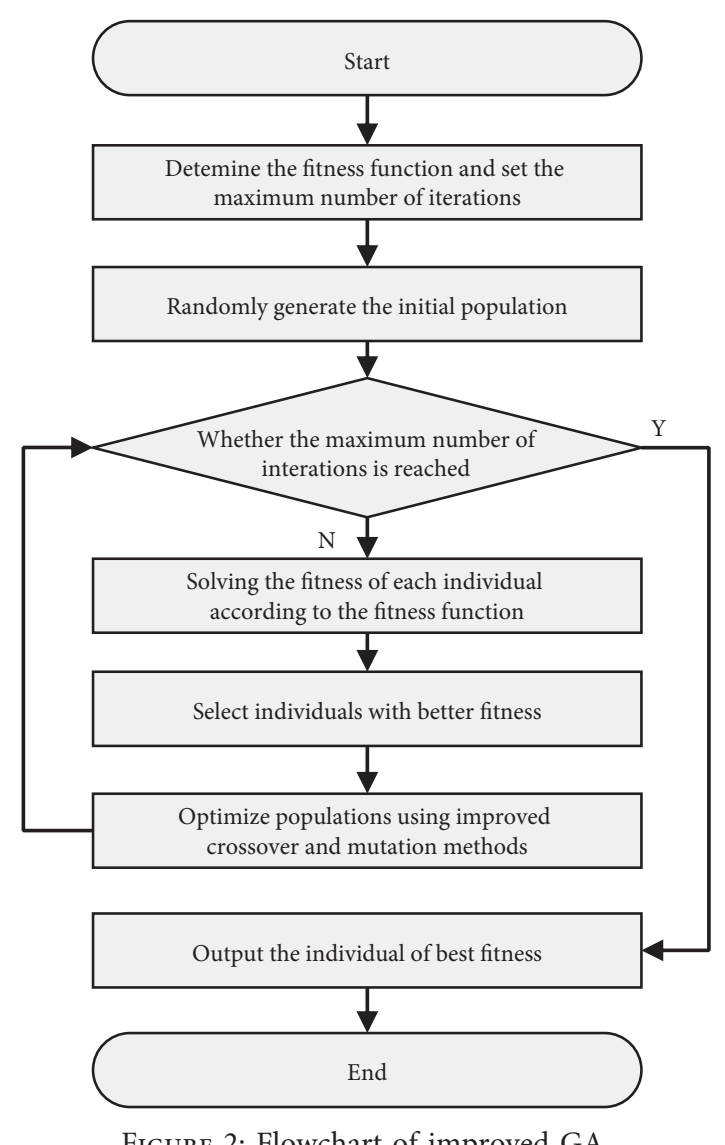

Figure 2: Flowchart of improved GA.

quantity range of containers is $10 \sim 500$, the range of QCs is $1 \sim 10$, the range of AGVs is $10 \sim 50$, and the range of ASCs is $1 \sim 10$. The weights in the objective function are $0.2,0.5$, and 0.3 , respectively. In order to reduce the random errors, I use the average value of 50 runs as the result of each ratio. The PGA is compared with traditional GA and particle swarm optimization algorithm (PSO), respectively [17]. The specific operation results are shown in Table 2.

It can be seen from Table 2 that the optimal fitness value is the largest when the ratio of QCs, AGVs, and ASCs is $1: 1$ : 1 in the case of the same number of containers. As the number of $\mathrm{AGVs}$ increases, the value of optimal fitness decreases gradually. When the ratio changes to $1: 2: 1$, the optimal fitness value changes greatly. When the ratio changes to $1: 3: 1$, the optimal fitness value does not change much. In theory, the scheme of $1: 3: 1$ should be adopted. In actual operation, as costs and economic benefits should also be considered, a scheme of $1: 2: 1$ should be adopted. Compared with other matching schemes, this scheme can not only ensure economic benefits, but also reduce the investigation and operation costs of QCs and ASCs, which is of great significance for the actual operation of the automated container terminal.

Figure 3 shows that in the case of the same number of containers, the running time of the algorithm increases gradually with the increase of the proportion. In the case of the same ratio, the solution time of algorithm increases as the number of containers is added and the increase is 
TABle 2: Experimental results.

\begin{tabular}{|c|c|c|c|c|c|c|c|c|}
\hline No. & $\begin{array}{l}\text { QC-AGV- } \\
\text { ASC }\end{array}$ & $\begin{array}{l}\text { Container } \\
\text { quantity }\end{array}$ & $\begin{array}{c}\text { Running time of } \\
\text { GA (s) }\end{array}$ & $\begin{array}{l}\text { Optimal } \\
\text { fitness }\end{array}$ & $\begin{array}{l}\text { Running time of } \\
\text { PGA (s) }\end{array}$ & $\begin{array}{l}\text { Optimal } \\
\text { fitness }\end{array}$ & $\begin{array}{l}\text { Running time of } \\
\text { PSO (s) }\end{array}$ & $\begin{array}{c}\text { Optimal } \\
\text { fitness }\end{array}$ \\
\hline 1 & $2-2-2$ & 100 & 2.08 & 976 & 1.69 & 874 & 1.73 & 895 \\
\hline 2 & $2-4-2$ & 100 & 2.64 & 476 & 2.20 & 436 & 2.34 & 452 \\
\hline 3 & $2-6-2$ & 100 & 3.38 & 378 & 2.71 & 353 & 2.82 & 376 \\
\hline 4 & $3-6-3$ & 100 & 3.92 & 295 & 3.26 & 227 & 3.34 & 239 \\
\hline 5 & $3-3-3$ & 200 & 5.58 & 907 & 4.92 & 899 & 5.11 & 904 \\
\hline 6 & $3-6-3$ & 200 & 6.63 & 541 & 5.13 & 483 & 5.20 & 490 \\
\hline 7 & $3-9-3$ & 200 & 7.56 & 412 & 5.45 & 327 & 5.49 & 325 \\
\hline 8 & $4-8-4$ & 200 & 8.27 & 498 & 5.83 & 445 & 5.92 & 456 \\
\hline 9 & $4-4-4$ & 300 & 8.99 & 1337 & 7.34 & 1285 & 7.47 & 1307 \\
\hline 10 & $4-8-4$ & 300 & 9.67 & 836 & 7.58 & 793 & 7.63 & 790 \\
\hline 11 & $4-12-4$ & 300 & 10.43 & 713 & 7.73 & 621 & 7.82 & 634 \\
\hline 12 & $5-5-5$ & 300 & 11.28 & 1463 & 7.52 & 1336 & 7.63 & 1362 \\
\hline 13 & $6-6-6$ & 400 & 14.15 & 1506 & 9.37 & 1397 & 9.45 & 1406 \\
\hline 14 & $6-12-6$ & 400 & 14.78 & 921 & 9.65 & 855 & 9.72 & 864 \\
\hline 15 & $8-16-8$ & 400 & 15.47 & 1007 & 9.89 & 928 & 9.93 & 943 \\
\hline 16 & $8-24-8$ & 400 & 16.23 & 857 & 10.23 & 764 & 10.44 & 782 \\
\hline 17 & $10-10-10$ & 500 & 19.92 & 1629 & 12.34 & 1580 & 12.53 & 1587 \\
\hline 18 & $10-20-10$ & 500 & 20.75 & 1148 & 12.72 & 1098 & 12.87 & 1102 \\
\hline 19 & $10-30-10$ & 500 & 21.63 & 898 & 13.13 & 853 & 13.26 & 862 \\
\hline 20 & $10-40-10$ & 500 & 22.29 & 632 & 13.58 & 573 & 13.64 & 591 \\
\hline
\end{tabular}

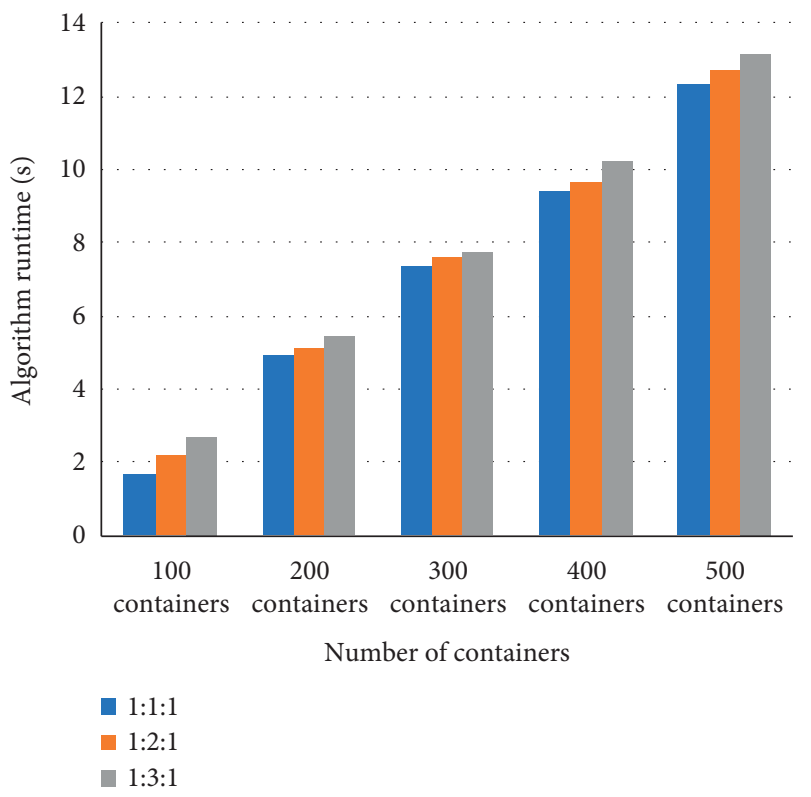

FIgURE 3: Algorithm representations of different container quantities.

significantly larger than that under the change of proportion. The main reason is that in the case of an increase in the ratio and the number of containers, the complexity of the problem increases gradually. In addition, the raise in the number of containers has a greater impact on the complexity, so the solution time is longer.

As can be seen from Table 2, the PGA runs obviously faster than traditional GA and slightly faster than PSO, which proves that the changes of the crossover and variation probability in real time effectively improve the running iteration of GA and PSO. Under the conditions that the number of containers is 100 and the number of QCs, AGVs, and ASCs are both 2, the comparison between the three iterations is made. The results of the comparisons between PGA, traditional GA, and PSO are shown in Figure 4. Figure 4 shows that PGA converges obviously faster than traditional GA and slightly faster than PSO. PGA and PSO find the optimal solution after about 30 iterations and GA needs more than 90 iterations, which indicates that the solving ability and speed of PGA are better.

To test the statistical significance of the differences between the population fitness values of PGA and GA, I performed one-way ANOVA tests [36]. The sixth column in Table 3 presents the computed $P$ value; it can be concluded 


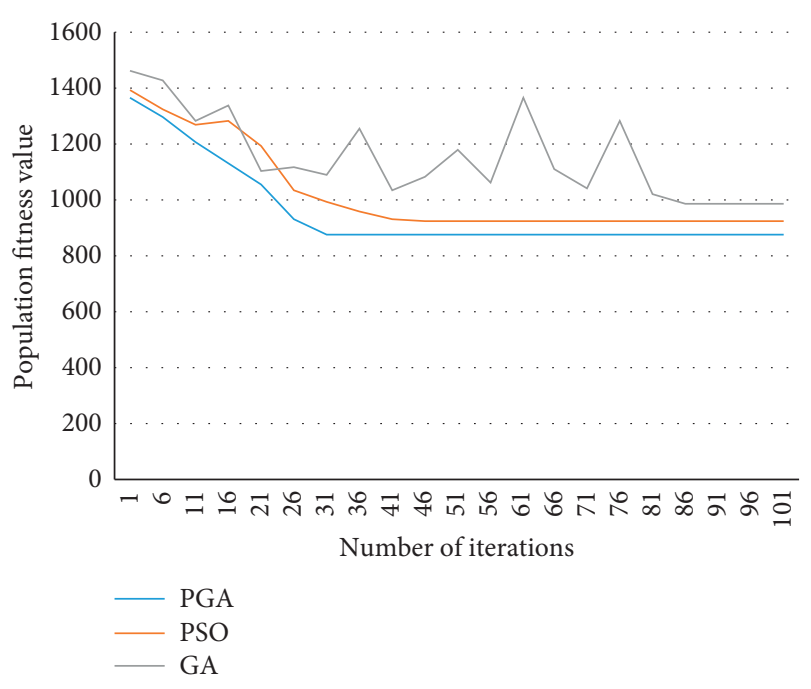

FIGURE 4: Comparison of iteration of three algorithms.

TABle 3: Significant differences between algorithms at 95 percent confidence level.

\begin{tabular}{lcccccc}
\hline $\begin{array}{l}\text { Difference } \\
\text { source }\end{array}$ & SS & df & MS & $F$ & $\begin{array}{c}P \\
\text { value }\end{array}$ & $F$ crit \\
\hline $\begin{array}{l}\text { Between } \\
\text { groups }\end{array}$ & 398677.7 & 1 & 398677.7 & 16.76133 & 0.0002 & 4.084746 \\
$\begin{array}{l}\text { In group } \\
\text { Total }\end{array}$ & 951422.8 & 40 & 23785.57 & & & \\
\hline
\end{tabular}

that the differences between the population fitness values of PGA and GA are statistically significant at a significance level of 0.05 .

\section{Conclusions}

According to the actual operation of the automated container terminal, I construct a three-stage integrated scheduling optimization model of the automated container terminal by studying the respective characteristics and internal relations of QCs, AGVs, and ASCs. The objective function is the minimum time for completing the entire loading and unloading tasks. Constraints are set according to the accrual operation of the automated container terminal. The improved GA, which is PGA, is used to solve the problem. Compared with traditional GA, the PGA with realtime crossover probability and variation probability can greatly improve the convergence speed and prevent the algorithm from falling into the local optimal solution. The experiments show the model constructed in this paper has an important reference to find the optimal ratio of the QCs, AGVs, and the ASCs at the automated container terminal, which is of great significance to improve the efficiency of the automated container terminal. Meanwhile compared with traditional GA, the PGA has a great improvement on solving speed and convergence.

We consider the three-stage integrated optimization of the automated container terminal scheduling. The optimization model in this paper does not consider the operations of dangerous goods or super-large containers. So, the scheduling for the cooperative optimization of these special containers will be the key and the direction for further study.

\section{Data Availability}

The data including models, tables, and figures which are used to support the findings of this study are included within the article.

\section{Conflicts of Interest}

The author declares that there are no conflicts of interest.

\section{Acknowledgments}

This paper was supported by Humanities and Social Sciences Research and Planning Fund/Youth Fund of the Ministry Education of China (18YJZCH116): Research on Integrated Optimization of Automated Container Terminal Scheduling under Uncertain Environment.

\section{References}

[1] X. Zhu and X. Li, "Iterative search method for total flowtime minimization no-wait flowshop problem," International Journal of Machine Learning and Cybernetics, vol. 6, no. 5, pp. 747-761, 2015.

[2] P. Legato, R. Trunfio, and F. Meisel, "Modeling and solving rich quay crane scheduling problems," Computers and Operations Research, vol. 39, no. 9, pp. 2063-2078, 2012.

[3] O. Uusal and C. Oguz, "Constraint programming approach to quay crane scheduling problem," Transportation Research Part E, vol. 59, pp. 108-122, 2013.

[4] J. H. Chen, D.-H. Lee, and M. Goh, "An effective mathematical formulation for the unidirectional cluster-based quay crane scheduling problem," European Journal of Operational Research, vol. 232, no. 1, pp. 198-208, 2014.

[5] Q. Q. Shao, Q. Xu, Z. Bian, and Z. H. Jin, "Stockpiling operating optimization for yard crane with containers delivery time uncertainty," Systems Engineering-Theory and Practice, vol. 35, no. 2, pp. 394-405, 2015.

[6] D. Chang, Z. Jiang, W. Yan, and J. He, "Developing a dynamic rolling-horizon decision strategy for yard crane scheduling," Advanced Engineering Informatics, vol. 25, no. 3, pp. 485-494, 2011.

[7] H. Javanshir and S. R. Seyedalizadeh Ganji, "Yard crane scheduling in port container terminals using genetic algorithm," Journal of Industrial Engineering International, vol. 11, no. 6, pp. 39-50, 2010.

[8] Q. C. Zeng, X. J. Zhang, W. H. Chen, and X. C. Zhu, "Optimization model for truck appointment based on BCMP queuing network," Journal of Systems Engineering, vol. 28, no. 5, pp. 592-599, 2013.

[9] Q. K. Cao and F. Zhao, "Port truck route optimization based on GA-ACO," Systems Engineering-Theory and Practice, vol. 33, no. 7, pp. 1820-1828, 2013.

[10] X. Guo, S. Y. Huang, W. J. Hsu, and M. Y. H. Low, "Dynamic yard crane dispatching in container terminals with predicted vehicle arrival information," Advanced Engineering Informatics, vol. 25, no. 3, pp. 472-484, 2011. 
[11] C. Ma and C. J. Liang, "Integrated quay crane allocation and yard truck schedule problem in container terminals," Journal of Guangxi University (Natural Science Edition), vol. 40, no. 3, pp. 643-650, 2015.

[12] T. B. Qin, H. Ge, and S. Mei, "Unidirectional quay crane scheduling problems solving by combination of mixed integer programming and constraint programming," Computer Integrated Manufacturing Systems, vol. 21, no. 2, pp. 546-555, 2015.

[13] Y. Q. Xu and X. L. Han, "United scheduling model optimization of yard truck, quay crane and yard crane," Journal of Chongqing Jiaotong University (Natural Science), vol. 32, no. 2, pp. 318-320, 2013.

[14] Y. Wu, J. Luo, D. Zhang, and M. Dong, “An integrated programming model for storage management and vehicle scheduling at container terminals," Research in Transportation Economics, vol. 42, no. 1, pp. 13-27, 2013.

[15] W. J. Mi, Y. F. Shen, and J. Wang, X. M. Yang, Collaborative decision-making on container pickup operation for gateway container selection and machinery resource allocation," Chinese Journal of Construction Machinery, vol. 12, no. 5, pp. 454-459, 2014.

[16] X. W. Xing, J. Mao, R. Zhang, and Z. H. Jin, “Optimization of container loading/unloading integrated scheduling in a container terminal based on hybrid flow shop," Chinese Journal of Management Science, vol. 22, no. 10, pp. 97-105, 2014.

[17] Y. Lu and M. Le, "The integrated optimization of container terminal scheduling with uncertain factors," Computers and Industrial Engineering, vol. 75, pp. 209-216, 2014.

[18] L. Chen, A. Langevin, and Z. Lu, "Integrated scheduling of crane handling and truck transportation in a maritime container terminal," European Journal of Operational Research, vol. 225, no. 1, pp. 142-152, 2013.

[19] S. Y. Zhang, Y. S. Yang, C. J. Liang, B. W. Xu, and J. J. Li, "Optimal control of multiple AGV path conflict in automated terminals," Journal of Transportation Systems Engineering and Information Technology, vol. 17, no. 2, pp. 83-89, 2017.

[20] R. Zaghdoud, K. Mesghouni, S. C. Dutilleul, K. Zidi, and K. Ghedira, "A hybrid method for assigning containers to AGVs in container terminal," IFAC-PapersOnLine, vol. 49, no. 3, pp. 96-103, 2016.

[21] J. Xin, R. R. Negenborn, F. Corman, and G. Lodewijks, "Control of interacting machines in automated container terminals using a sequential planning approach for collision avoidance," Transportation Research Part C: Emerging Technologies, vol. 60, pp. 377-396, 2015.

[22] J. Luo, Y. Wu, and A. B. Mendes, "Modelling of integrated vehicle scheduling and container storage problems in unloading process at an automated container terminal," Computers and Industrial Engineering, vol. 94, pp. 32-44, 2016.

[23] C. Wei, Z. H. Hu, C. F. Gao, and X. J. Luo, "Scheduling model and algorithm of twin synchronized stacking cranes in stack yard of automated container terminal," Journal of Dalian Maritime University, vol. 41, no. 4, pp. 75-89, 2015.

[24] S. Y. Ma, Y. S. Yang, and C. J. Liang, "Collaborative scheduling of double-trolley quay cranes and AGV based on PSO at automated container terminal," Computer Applications and Software, vol. 35, no. 10, pp. 17-22, 2018.

[25] Y. S. Yang, Y. Y. Feng, C. J. Liang, B. W. Xu, and J. J. Li, "Integrated scheduling of automated guided vehicles and railmounted gantry cranes at automated container terminals,"
Journal of Shanghai Maritime University, vol. 38, no. 2, pp. 1-6, 2017.

[26] M. L. Le and Q. B. Zhang, "Integrated scheduling of quay cranes, automated guided vehicles and automated yard cranes at automated container terminals," Journal of Qingdao University of Science and Technology (Natural Science Edition), vol. 36, no. 5, pp. 569-574, 2015.

[27] Q. Zeng and Z. Yang, "Integrating simulation and optimization to schedule loading operations in container terminals," Computers and Operations Research and Operations Research, vol. 36, no. 6, pp. 1935-1944, 2009.

[28] S. L. Hu and Z. H. Hu, "Full freedom optimization for integrated quay cranes, trucks and yard cranes in container port," Journal of Wuhan University of Technology, vol. 34, no. 5, pp. 58-63, 2012.

[29] H. Dkhil, A. Yassine, and H. Chabchoub, "Optimization of container handling systems in automated maritime terminal," Advanced Methods for Computational Collective Intelligence, vol. 457, pp. 301-312, 2013.

[30] J. H. Holland, Adaptation in Natural and Artificial Systems, Ann Arbor University of Michigan Press, Ann Arbor, MI, USA, 1975.

[31] J. Zhu, X. Li, and W. Shen, "Effective genetic algorithm for resource-constrained project scheduling with limited preemptions," International Journal of Machine Learning and Cybernetics, vol. 2, no. 2, pp. 55-65, 2011.

[32] S. Khalilpourazari and S. Khalilpourazary, "SCWOA: an efficient hybrid algorithm for parameter optimization of multipass milling process," Journal of Industrial and Production Engineering, vol. 35, no. 3, pp. 135-147, 2018.

[33] Y. Wang, X. Jiang, L. H. Lee, E. P. Chew, and K. C. Tan, “Tree based searching approaches for integrated vehicle dispatching and container allocation in a transshipment hub," Expert Systems with Applications, vol. 74, pp. 139-150, 2017.

[34] I. Bavarsad Salehpoor and S. Molla-Alizadeh-Zavardehi, "A constrained portfolio selection model at considering riskadjusted measure by using hybrid meta-heuristic algorithms," Applied Soft Computing, vol. 75, pp. 233-253, 2019.

[35] H. Hu, X. Chen, T. Wang, and Y. Zhang, "A three-stage decomposition method for the joint vehicle dispatching and storage allocation problem in automated container terminals," Computers and Industrial Engineering, vol. 129, pp. 90-101, 2019.

[36] S. Khalilpourazari, S. H. R. Pasandideh, and S. T. A. Niaki, "Optimization of multi-product economic production quantity model with partial backordering and physical constraints: SQP, SFS, SA, and WCA," Applied Soft Computing, vol. 49, pp. 770-791, 2016. 